\title{
Lymphangioma of the spleen in an asymptomatic patient
}

\author{
Chandrasinghe P.C, $\mathrm{MBBS}^{1}$, MarasingheN.C, $\mathrm{MBBS}^{1}$, Abeyrathne I.G.K.P, MBBS ${ }^{1}$, \\ Fernando R.F, MBBS, MS, FRCS ${ }^{2}$ \\ 1 Surgical registrar, University Surgical Unit, North Colombo Teaching Hospital, Ragama, Sri Lanka. \\ 2 Professor in Surgery, University Surgical Unit, North Colombo Teaching Hospital, Ragama, Sri Lanka.
}

Key words: Splenic cyst; Lymphangioma.

\section{Introduction}

Splenic cysts due to non parasitic causes are rare [1]. Lymphangioma is a very uncommon cause for benign cysts of the spleen. It is a benign neoplasm of the lymphatic system mainly seen in children and is rare in adults $[2,3]$. Perusal of literature did not reveal any report of lymphangioma of the spleen in Sri Lanka.

\section{Case report}

A 42 year old female was detected to have an abdominal mass at a routine medical examination. She was referred to a surgeon for further investigations. She had no history of parasitic infection or foreign travel. Examination revealed a mass in the left hypochondria, consistent with an enlarged spleen. She was found to have an enlarged spleen on ultrasonography and a CT scan of the abdomen revealed a large multi cystic lesion in the spleen. The cysts were well circumscribed and peripheral calcifications were seen. Her serum investigations were unremarkable. Possible differential diagnoses entertained were benign cyst or hydatid cyst. She underwent total splenectomy with an uneventful recovery. The histopathology report confirmed a multiple cystic lymphangiomas of the spleen with little residual splenic parenchyma.

\section{Discussion}

With lymphangiomatosis, the spleen is diffusely replaced by expanding lymphangiomas that leave little remaining splenic parenchyma. Cystic

Correspondence: Ranil Fernando, Professor in Surgery, University Surgical Unit, North Colombo Teaching Hospital, Ragama, Sri Lanka.

Email: ranilfern@sltnet.lk lymphangioma is a benign neoplasm composed of a malformation of the lymphatic system $[2,3]$. They are generally seen in the neck (75\%) and axilla (20\%) in children less than 2 years of age [4]. Less common sites are the mediastinum, retroperitoneum, extremities and viscera [5]. There is no difference in the incidence between the sexes [3]. Amongst primary benign neoplasms of the spleen, hemangioma is the most common while the rare ones, apart from lymphangioma, include hamartoma and littoral cell angioma [5]. Clinical presentation could vary from an incidental finding in an ultrasound scan to having left upper quadrant pain, nausea and abdominal distention. Symptoms are closely related to the size of the cyst. The largest cyst in this patient was $7 \mathrm{~cm}$ in diameter. The complications associated with extensive or larger lymphangiomas of the spleen include bleeding, consumptive coagulopathy, hypersplenism, and portal hypertension [6] none of which were seen in this patient. On sonograms, these cystic lesions appear as well-defined hypoechoic masses with occasional internal septations and intralocular echogenic debris [6]. On CT scans, lymphangiomas appear as single or multiple thinwalled low-attenuation masses with sharp margins that are typically subcapsular in location. No significant contrast enhancement is typically seen. The presence of curvilinear peripheral mural calcifications suggests the diagnosis of cystic lymphangioma [7] which could be seen in this patient's CT scan (Fig 01). Splenic lymphangiomas are classified as capillary, cavernous and cystic [4]. Our patient had the cystic type. They consist of a single layer of flattened endothelium-lined spaces, which are filled with eosinophilic proteinaceous material instead of blood as seen in hemangiomas.

The Sri Lanka Journal of Surgery 2011; 29(2):100-102. 


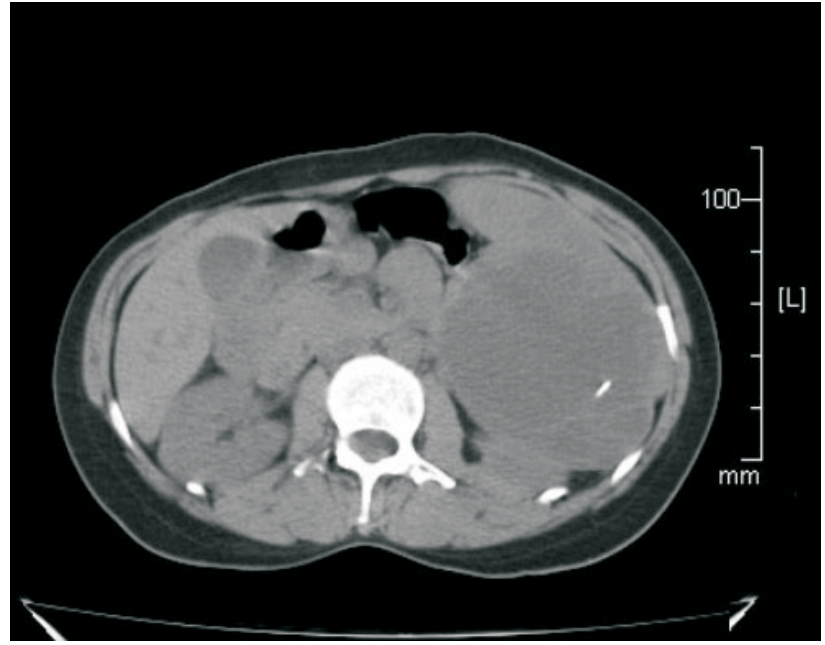

Figure 1.CT scan of the abdomen showing a cystic enlargement of the spleen.

When the histologic characteristics are not clear, the endothelial origin of the cyst may be established with immunohistochemical techniques that demonstrate reactivity for factor VIII, a finding that confirms the diagnosis of lymphangioma [8]. Surgical resection is indicated to prevent complications such as infection, rupture, and bleeding. Total splenectomy has been the common practice in the past [3]. Conservative treatment with aspiration, drainage and sclerosis, has a high risk of recurrence. Partial splenectomy is currently recommended for small lesions when technically to preserve splenic tissue as much as possible. The post-operative recurrence rate is low [10]. There have been very few reports of a splenic lymphangioma developing into malignant lymphangiosarcoma [9].

\section{References}

1. Liew SH, Clements WD, Wilson BG. Splenic conservation in the management of large splenic cysts: case report and literature review. Journal of Royal College of surgeons, Edinburgh. 1997; 42(2):135-7.

2. Kim DH, Byun JN, Jang JY. Cystic lymphangioma involving the mesentery and the retroperitoneum: A case report. Journal of the Korean Radiological Society.2005; 52:347-350.

3. Chung SH, Park YS, Jo YJ, Kim SH, Jun DW, Son BK, Jung JY, Baek DH, Kim DH, JungYY, Lee WM. Asymptomatic lymphangioma

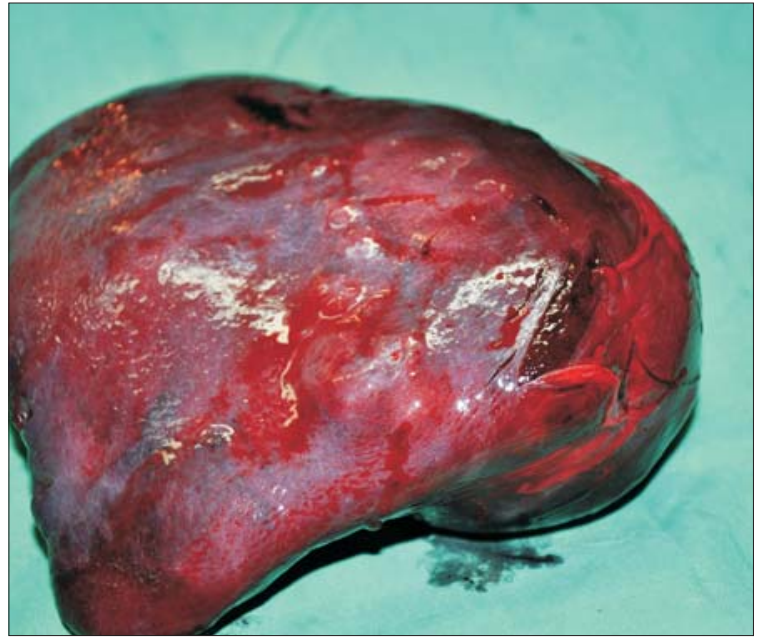

Figure 2. Excised spleen with cystic enlargement.

involving the spleen and retroperitoneum in adults: World Journal of Gastroenterology. 2009; 15(44): 5620-5623.

4. Abbott RM, Levy AD, Aguilera NS, Gorospe L, Thompson WM. From the archives of the AFIP: primary vascular neoplasms of the spleen: radiologic-pathologic correlation. Radiographics 2004; 24:113763.

5. Komatsuda T, Ishida H, Konno K, et al. Splenic lymphangioma: US and $\mathrm{CT}$ diagnosis and clinical manifestations. Abdominal Imaging 1999; 24:414-417.

6. Urrutia M, Mergo PJ, Ros LH, Torres GM, Ros PR. Cystic masses of the spleen: radiologicpathologic correlation. Radiographics 1996; 16:107-129.

7. Pistoia F, Markowitz SK. Splenic lymphangiomatosis: CT diagnosis. American Journal of Roentgenology 1988;150:1212.

8. Enghardt MH, Allegra SR. An immunohistologic study of a splenic cyst. Military Medicine. 1987; 152:321-323.

9. Feigenberg Z, Wysenbeek A, Avidor E, Dintsman M. Malignant lymphangioma of the spleen. Israel Journal of Medical Sciences.1983; 19:202-204.

10. Chang CH, Hsieh CB, Yu JC, Jan CI. A case of lymphangioma of the spleen. Journal of Medical Sciences.2004; 24:109-112. 
The Sri Lanka Journal of Surgery 2011; 29(2)

\section{Key Learning Points}

$<$ Lymphangioma of the spleen is a rare entity which could become large but remain asymptomatic.

$<$ Radiological investigation of choice is a contrast enhanced CT scan.

$<$ Splenic conservation is currently practised when excising a splenic cyst. 